\title{
Smart energy households' pilot projects in The Netherlands with a design-driven approach
}

\author{
Daphne Geelen ${ }^{1}$, Arno Scheepens ${ }^{1}$, Charlotte Kobus ${ }^{1}$, Uchechi Obinna ${ }^{1}$, \\ Ruth Mugge ${ }^{1}$, Jan Schoormans ${ }^{1}$, Angele Reinders ${ }^{1,2}$ \\ ${ }^{1}$ Faculty of Industrial Design Engineering \\ Delft University of Technology \\ Delft, The Netherlands \\ ${ }^{2}$ Faculty of Engineering Technology \\ University of Twente \\ Enschede, The Netherlands
}

\begin{abstract}
Residential smart grid projects can be evaluated by a design-driven approach, which focuses on gaining insights for successful product and service development by taking the endusers as a starting point. Because only little experience exists with this design-driven approach, this paper addresses how four pilot projects with smart energy households in the Netherlands namely (A) Power Matching City, (B) Smart Wash, (C) Your Energy Moment and (D) Nieuwveense landen - are being or will be evaluated. The pilot projects illustrate that by studying endusers' perception of and interaction with technology, insights are created that can be used for development of product and service development, not only from a technical point of view but also in terms of financial and non-financial value creation. A design-driven approach can therefore help to broaden the focus of product and service development from mere technical feasibility to a multidisciplinary exploration of how to create successful products and services for smart grid households.
\end{abstract}

Index Terms - End-users, Design, Pilot Project, Pricing, Smart energy households

\section{INTRODUCTION}

What lessons can be learnt from analyzing current residential smart grid projects from a design perspective, in particular with regards to the performance and development of products and services offered to end-users? This is an interesting research question for which an answer can support the successful deployment of future residential smart grids, also called smart energy households. At present, smart grid pilot projects at the neighborhood or household level, seem to be a top-down effort, driven by the technical implementation of systems that balance energy demand and supply. However, domestic demand response involves adoption of new technology and behavioral change by residential end-users. Households will only change behavior or adopt new technologies when they can recognize and appreciate the added economic as well as the non-economic value. Innovation therefore requires a more multi-faceted approach. It is essential to be aware of the context for which technology is developed and to understand underlying needs and motivations of end-users. This means that for successful deployment of domestic smart energy systems a design-driven approach might be important. The overall aim of design research is to enable the development of more successful products and services [1], in other words: to create value for end-users. A design-driven approach is multidisciplinary, integrating knowledge from for example engineering, natural, human and cultural sciences.

An inventory of smart grid projects worldwide by [2] revealed about 49 world smart grid projects at the household level, most of which are still ongoing. The completed projects at this level generally involve the installation and testing of smart meters and advanced metering infrastructure. Current approaches to smart grid deployment tend to be based on technology, and the changing of end-user behavior in order to optimize the use of this technology. An integrated approach that combines top-down implementation with end-users' needs and perceptions, or the so called bottom-up requirements, could fill this gap. Such an approach might be necessary in order to effectively develop new smart grid- related products and services that will be successfully adopted by end-users. This is what a design-driven approach might offer.

Only little experience exists with a design-driven approach in smart grid pilots. On the one hand this is due to the relatively young research field of smart grids, on the other hand because of the strong focus on technology implementation in this field. Therefore, we will discuss in this paper how four different pilot projects with smart energy households in the Netherlands have been analyzed with design-driven research methods, what can be learned from these experiences and what could be the benefits of a designdriven approach on top of research with a technical scope for smart grid deployment.

\section{The SMART ENERGy Households PROJECTS}

The pilot projects in which a design-driven approach was used are: (A) Power Matching City, (B) Smart Wash, (C) Your Energy Moment and (D) Nieuwveense landen. The projects are all located in The Netherlands.
Sponsors and partners of the projects presented in this paper are: Agentschap NL, IPIN of the Dutch ministery of Economic Affairs. Enexis. Essent, Gasunie, KEMA DNV, and Energy Valley. Flexicontrol, DONG Energy, GreenChoice, CGI, SWZ and TU Eindhoven. MeppelEnergie, Gemeente Meppel, EnergyValley, iNRG, Rendo Duurzaam, Universiteit Twente, Delft University of Technology. 


\section{A. Power Matching City}

The Power Matching City demonstration project is one of the first projects to implement smart energy technology in households connected through a smart-grid. The first phase of the project started in 2007: A local smart grid with 22 households was realized. The households were equipped with a hybrid heat pump or a micro-cogeneration unit, both in combination with hot water tanks. In twelve of the households smart dishwashers and washing machines were installed. Furthermore, each household generates PV-solar energy, either by their own PV system or through virtual coupling of a PV system located on the building of the project partners. The energy flows in the local smart grid are managed via 'PowerMatcher', an agent-based algorithm that, based on market mechanisms and user settings, controls when the heat pumps, micro-cogeneration units, dish washers and washing machines are switched on and off. The end-users could access information about their energy consumption and production via Internet by logging in to a 'user portal'. The complete setup of PowerMatching City is described in more detail in [3] and [4].

Over the course of the demonstration project, after technical feasibility had been demonstrated, ways to involve the residential end-users were explored, see [5] and [6]. Qualitative research was conducted to evaluate the experiences and expectations of the end-users for the smart energy system. This research was executed with interviews, questionnaires, focus groups and co-design sessions. Compared to the interviews, questionnaires and focus groups, the co-design sessions were used as a more active way to elicit experiences as well as to generate ideas to shape the interaction between end-users and the implemented technology. Additionally, quantitative analysis of the energy consumption and production took place in order to gain insight in the balance between production and consumption in the cluster in relation to the energy delivered from the grid to the cluster and vice versa. Measurements of energy consumption and production in the households at 5 minutes intervals were used, taken from April $1^{\text {st }}, 2012$ to January $31^{\text {st }}$, 2013. These data were aggregated to provide cluster level information. Indicators were used to evaluate the energy balance in the cluster describing (1) electricity production in the cluster compared to energy consumption, (2) the amount of consumption from production in the cluster compared to the total production in the cluster and (3) the amount of consumption from production within the cluster compared to overall consumption.

With regard to the experiences of the end-users, satisfactory comfort levels were reported for the heating systems. This was a boundary condition in the technology development and implementation. However, several end-users indicated to lack a sense of insight and control that enables them to understand how the system operates and to reach their households' energy related goals. These goals include energy saving, use of renewable energy and trading energy. Additionally the households do not perceive that they are part of a smart grid where energy production and consumption is balanced in the PowerMatching City network. The goals as well as the extent to which end-users would like to be enabled to actively change their behavior differ per household.

The results also indicate that in addition to information at household level, information at community level would be interesting for the end-users to (1) support reaching one's goals (by comparison of energy use and by peer-learning concerning practices/tips) and (2) see the relations of household energy consumption and production with energy flows within the entire cluster and when possible act on this information. Further research is required to confirm these findings.

Concerning the energy balance analysis, most of the energy that is produced in the cluster was found to be consumed in the cluster. This balance is a bit 'off' in winter, because most of the energy production came from the PV solar panels in the period that was analyzed. Most of the energy needs are still covered by purchasing electricity from the grid. Increased renewables capacity could solve this (if desired). Also changes on the end-user side are to be considered, such as energy efficiency measures and behavioral changes to save energy and shift loads (in addition to the automated shifting). For this, the end-users would need to be provided with actionable information as well as means to manage energy consumption and production in their home.

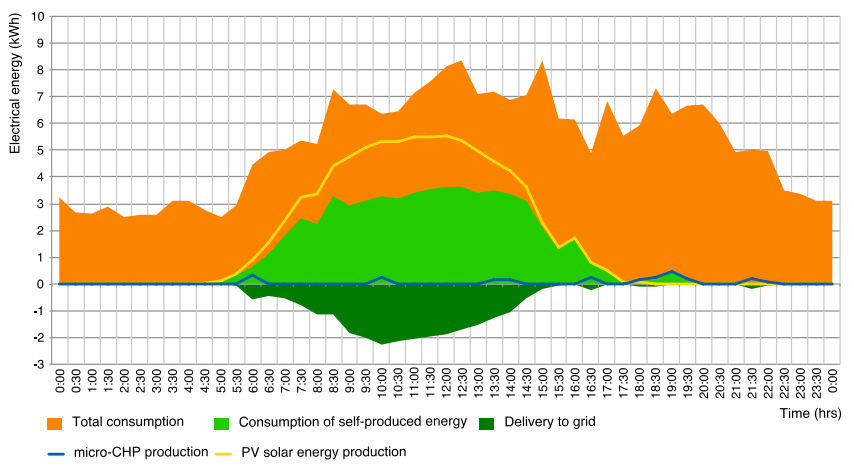

Figure 1. Daily patterns of electrical energy consumption and production in the Power Matching City pilot project in August $19^{\text {th }}, 2012$. (Sunday)

The results of the research were used for the second phase of the project, in which an additional 18 households are connected in the smart grid. End-users are provided with an in-home energy display with improved feedback and control to enable demand response by behavioral changes on top of the automatic demand response operating in the background. Additionally, two business propositions will be tested: one optimizing for use of locally produced energy and one optimizing for lowest costs. The algorithm of PowerMatcher is set to enable these two propositions.

\section{B. Smart Wash}

The pilot project Smart Wash that was conducted in 2011, examined the factors that influence the likelihood that people will shift their electricity consumption to other moments in time to match sustainable supply [7]. The 24 households involved in this study were equipped with a system consisting of solar panels, a smart meter, a smart washing machine and an Energy Management System (EMS). The EMS collects consumption data from the smart meter and production data 
from the solar panels. These insights are given real-time, but also presented to users in an historical overview. Feed-forward is given in the form of hourly solar radiation forecasts. There was no financial incentive. The washing machine operates like a normal washing machine, except from the fact that the user can decide when the smart washing should be finished and after putting in the laundry, it will receive a best moment to start from the EMS. When needed, users were able to overrule the system.

According to [8] it is important to investigate beyond the kWh's to uncover how and for whom the interventions worked. Hence, washing machine consumption data and production data were analyzed. Furthermore, twenty-one semi-structured interviews were conducted with households who had used the EMS 'Smart Wash' for several months. Users were asked whether and how they matched their electricity consumption to the availability of local solar electricity. Users also explained why and how they made use of the feedback and feed-forward that was provided. Also, they explained why they did (not) use the system's feature to operate the washing machine autonomously. Users were asked to describe the system variables that supported and disturbed new behaviour. Last, users were asked why and to what extent users felt motivated to perform the new behaviour. Various factors related to the household were discussed that influenced behaviour change. It provided rich insights. These insights resulted in several recommendations for the future design of EMSs.

First, as also found in energy reduction interventions with EMSs, giving real time and historical feedback on energy consumption and production is necessary. Due to feedback, participants felt more in control over their own electricity consumption. Another form of feedback that facilitated shifting electricity usage of the washing machine was the scoring of 'green washes' (a point for every wash cycle in hours of sun shine). Users reported that this reward system made it fun and challenging and it felt like an acknowledgement for their effort. Besides feedback, feedforward on the expected solar radiation is considered a significant system variable for shifting consumption.

The autonomy of the smart washing machine was not always used. When a user is home during the day, advanced (and often more expensive) technology is not always needed. Although following the simple rule 'washing when the sun is shining' might not be the optimum; it is still valuable for peak reduction. Nevertheless, the system's ability to work autonomously was appreciated by users who are not at home during the day. This finding stresses the importance of being context sensitive and to define different segments when developing smart grid products and services. Furthermore, users appreciated the overrule option to remain in control of a particular event.

An unexpected effect, but interesting for future design of EMSs was users' goal setting to reach 'zero'. Some users balanced production and consumption precisely by turning on other appliances (e.g., dishwasher) for which usage was already planned. It is recommended to facilitate this type of goal setting when developing new interfaces for EMS.
An option that users were missing in this EMS was to compare their performance with other smart washers (e.g. ranking). More abstract system variables to take into account when designing EMSs are complexity and trustworthiness. When users consider the system as difficult to use or untrustworthy, it disturbs interaction. It results in frustration and in some cases, the discontinuation of using (parts of) the system and rejecting its advice. Therefore it is very important to test technology by usability tests on these aspects before full deployment.

\section{Your Energy Moment}

Your Energy Moment are pilot projects on Smart Grids with households in Breda and Zwolle in the Netherlands. In these newly built districts, residents produce solar energy, are equipped with a smart meter, an Energy Management System (EMS) and a smart washing machine, which can communicate with the EMS. The insights from Smart Wash were used to further develop the technology for Your Energy Moment. Unlike Smart Wash, these participants also receive dynamic prices. The objective is to investigate if households are able and willing to adapt their demand to times on which supply is abundant. And of course, who will and who will not. Furthermore, it is particularly interesting how participants use and perceive the offered technology and dynamic prices. New design recommendations must result from these pilots to enable the development of more successful products and services. The pilot projects will be in operation for 24 months and have started in November 2012 (Zwolle, $\mathrm{n}=104,97 \%$ response) and March 2013 (Breda, $n=148$, 66\% response). Participants can use the washing machine or other domestic appliances at times when it is beneficial for the environment (when the sun is shining), or when costs of energy are low. Participants receive information on preferred moments to use energy 24 hours ahead in blocks of two hours on their EMS. They can choose to see local production forecasts or tariffs and the scheduling algorithm for the smart appliances will optimize accordingly. Next to forecasts, feedback is given showing the amount of energy used and especially, when it was used. Before installation, all participating households had to fill in a questionnaire to map household characteristics, expectations and intentions. Now the technology is installed in the homes, a small group will be interviewed and all participants will have to fill in the questionnaires periodically. Questionnaires entail (amongst others) intended and reported behavior, attitude towards the concepts (e.g. ease of use and usefulness motivation to participate and to change demand, routine formation, reported influence of design elements on behavior and advice for new attributes. Furthermore, interactions with the energy display and with the smart appliances will be monitored to analyze how the technology is used. Additionally, smart meter data, washing machine (and heat pump in some cases) consumption data and solar production data will be analyzed and compared to a reference group to quantify results.

Taken together, insights must be gathered (1) to quantify whether households are able and willing to shift demand (2) to develop new design recommendations for smart grids for households and (3) to give insights for segmentation of households for smart grids. 


\section{Nieuwveense landen}

In Meppel, a new residential area (Nieuwveense Landen) is to be built within the next two decades (-2030). To provide these future households with affordable and responsible heating and cooling, a new energy company has been created. The company plans to link biogas production from a wastewater treatment plant through CHP generation with underground heat and cold storage systems (heat pumps) as well as heat exchanger systems to deliver the heat \& cold to the end-users, using the produced electricity to operate the system. The focus is on creating a Product-Service System (PSS): selling heat \& cold instead of products, services and, in this case, natural gas.

The Faculty of Industrial Design Engineering is involved in this project to study the consumer-side of this smart grid heating system. Consumer acceptance is an important factor for the design of complex smart energy PSS, since successful market implementation of smart grid systems is crucial to obtain access to its tentative advantages. The proposed PSS business model for selling heat (and cold) instead of a mix of products, services and natural gas is new to consumers, and it will be investigated how consumers respond to such offerings in the market. Therefore the primary aim of this research is to explore to what extent end-users are willing to accept the proposed PSS relative to incumbent offerings in the market.

Most research activities in smart-grid projects involving end-users seem to be directed towards aiding or motivating the end-users to change their behavior to achieve energy reduction and peak shaving and/or shifting. The underlying assumption is that end-users want to save energy and thereby also costs, and are willing to put in the extra effort. However, these "extra" activities, relative to the incumbent systems, seem to be somewhat of a paradox, since more user involvement might decrease the experienced comfort of the average consumers in household settings. As shown by [9], around $24 \%$ of the Dutch households never look at, or alter the settings on their thermostat. On top of that, around half of the Dutch residents are unaware of the height of their energy costs, even though these correspond with around $8 \%$ of the average household income. They conclude: "Pre-programmed, smarter temperature control devices would be an obvious solution, comparable to automated enrolment into retirement saving programs."

PSS business models have been widely discussed as having potential for environmental impact reduction whilst creating value for its end-users, as is also the case with the proposed PSS at Nieuwveense Landen. Therefore the additional aim of our research is also to evaluate the proposed system design on basis of the Ecocosts-Value Ratio (EVR) [10], including the design of the buildings the PSS is implemented in. The EVR model is applied to ensure that the new PSS not only has a lower environmental impact, but is also more desirable than the offerings currently in the market (eco-efficient value creation).

The pursuit of the double objective of eco-efficient value creation leads to competitive, environmentally less unsustainable PSS designs which are to be tested in 4 pilot houses at the end of 2013. In these pilot houses, 4 different
PSS designs are tested and evaluated according to the EVR model, ranging from an autonomous, self-learning system to a system where full responsibility for optimal operation is left in the hands of the consumer. The main hypothesis is that autonomous, self-learning systems are better able to decrease the environmental impacts whilst maintaining or even improving comfort, and thereby acceptance of such PSS. The PSS can thereafter be optimized according to the findings and implemented in the further development of the residential area of Nieuwveense Landen in Meppel for different types of endusers.

\section{DISCUSSION AND CONCLUSION}

Based on the descriptions of the projects in the previous section, it can be observed that the research in each pilot project is different in terms of the technologies, involvement of end-users and research questions and approaches. A general denominator is that the end-user is an important part of starting point for evaluation of the smart energy products and services that are implemented. Furthermore, a joint goal is to gain insight for future product and service development.

The design-driven approach in the pilot project addresses the interaction between end-user and technology. The research evaluates how end-users use the products and services, how end-user perception, and if and how behavior is affected by the system design. The underlying thought is that end-users have to be willing to accept new technology and new habits. As we saw in Smart Wash, end-users were changing their behavior relatively easily, while in PowerMatching City, where behavior change was not intended, end-users actually indicated that they needed support in changing their behavior. The way in which the interaction between technology and end-users were shaped, influenced the effects on behavior, and satisfaction with the products and services that were offered.

The involved research methods in the pilot projects include questionnaires, interviews, focus groups, usability research and co-creation. These methods are complemented with evaluations of measured data on energy consumption, production and eventually trading. Co-creation activities, i.e. end-users take part in design and evaluation of products and services, can be used. These methods provide deeper insight than methods, which require less end-user involvement such as interviews and questionnaires.

For all four projects, the results are or will be used to formulate design recommendations, which often are directly applied in a next phase of the project. The results from the first phase of Powermatching City are used for development of propositions for the second phase, Smart Wash results were fed into Your Energy Moment and the research described for Nieuwveense Landen is used for PSS design towards large scale implementation. In all cases the findings would affect the product and service design in terms of core technical functioning, e.g. the mechanisms for supply and demand matching, as well as user behavior and perception related aspects, such as user interfaces.

From a design perspective, involvement of end-users is key in order to know what to design and how to match technology to end-users. With a dominantly technical 
approach, a system may be built that functions according to specifications, but when it does not match the end-users' demands sufficiently, it will not be accepted or the potential of a technology may not be reached. As for example in Smart Wash, when users considered the system as difficult to use or untrustworthy, the use of (part of) the system was discontinued and advice rejected. Similarly, in PowerMatching City, a lack of energy feedback inhibited endusers to adjust their behavior to contribute to energy management in the cluster.

With a design-driven approach the design of the products and services for households is approached as a multidisciplinary design problem, aiming to match technical potential with end-user demands, as to create value for the end-user, both economic as non-economic. Thereby, it is expected that in this way products and services can be created that will be accepted by end-users and simultaneously are able to reach their full potential for both end-user and energy system. As the case of Nieuwveense Landen demonstrates, rather than providing technological means to end-users, the product-service-system approach prioritizes the delivery of a service, namely heat at lowest environmental cost and optimal value for its end-users.

Taking the end-users' interests and abilities as a starting point, development of technical systems that are sub-optimal from the perspective of the end-users and therefore not accepted or used to their full potential can be avoided. A design-driven approach may even speed up the process of product \& service development as it addresses what end-users will find acceptable, be willing to use an pay for on top of technical feasibility.

The ability of the EVR model to evaluate the degree of eco-efficient value creation of smart grid offerings is highly relevant, since it simultaneously assesses the value for the end-users which ensures profit for the providers of the PSS, as well as its potential for environmental impact reduction which is only achieved in practice by ensuring it is a desirable offering.

To conclude, a design-driven approach can beneficial for the development and implementation of smart grid products and services, since it takes end-users as a starting point, who will ultimately determine the success of smart grid projects. This approach can help to broaden the focus from a separated approach to technological feasibility and end-user behavior to a multidisciplinary exploration of how to involve end-users in balancing supply and demand with smart grids.

\section{REFERENCES}

[1] L.T.M. Blessing \& A. Chakrabarti, "DRM, a Design Research Methodology", Springer, 2009, ISBN 978-1-84882-586-4

[2] U. Obinna , P. Joore, L. Wauben, A. Reinders, "Water related sustainable innovations and the smart grid", in Proceedings of the 16th European Roundtable on Sustainable Consumption and Production (ERSCP), 2013

[3] F. Bliek, A. van den Noort, B. Roossien, R. Kamphuis, J. de Wit, J. van der Velde, and M. Eijgelaar, "PowerMatching City, a living lab smart grid demonstration," Innovative Smart Grid Technologies Conference Europe (ISGT Europe), 2010 IEEE PES. pp. 1-8, 2010.

[4] K. Kok, B. Roossien, P. MacDougall, O. van Pruissen, G. Venekamp, R. Kamphuis, J. Laarakkers, and C. Warmer, "Dynamic pricing by scalable energy management systems - Field experiences and simulation results using PowerMatcher," in 2012 IEEE Power and Energy Society General Meeting, 2012, pp. 1-8.

[5] D. Geelen, A. Reinders, and D. Keyson, "Involving end-users in smart grid technology: A community-based approach for behaviour change," in Diversity and unity: Proceedings of IASDR2011, the 4th World Conference on Design Research, 2011.

[6] D. Geelen, A. Reinders, and D. Keyson, "Empowering the end-user in smart grids: Recommendations for the design of products and services," Accepted for publication in Energy Policy, 2013

[7] Kobus, C. B. A, R. Mugge \& J. P. L. Schoormans, "Washing when the sun is shining! How users interact with a household energy management system." Ergonomics pp. 1-12 2012.

[8] Karlin, B. \& Ford, R. "Beyond kWh: A New Tool for Assessing Behavior-Based Energy Efficiency Programs." Proceedings of the International Energy Program Evaluation Conference (IEPEC). In press.

[9] Brounen, D., Kok, N., Quigley, J.M., "Energy literacy, awareness, and conservation behavior of residential households", Energy Economics 38, p.p. 42-50, 2013.

[10] Vogtländer J.G., Baetens, B., Bijma, A., Brandjes, E., Lindeijer, E., Segers, M., Witte, F., Brezet, J.C., Hendriks, Ch.F, "LCA-based assessment of sustainability: the Eco-costs/Value Ratio (EVR)", VSSD: Delft, the Netherlands, 2010 